\title{
Rates of convergence of lognormal extremes under power normalization
}

\author{
Jianwen Huang ${ }^{1 *}$, Shouquan Chen² and Yanmin Liu
}

"Correspondence:

hjw1303987297@126.com

'School of Mathematics and

Computational Science, Zunyi

Normal College, Zunyi, 563002,

China

Full list of author information is

available at the end of the article

\begin{abstract}
Let $\left\{X_{n}, n \geq 1\right\}$ be an independent and identically distributed random sequence with common distribution $F$ obeying the lognormal distribution. In this paper, we obtain the exact uniform convergence rate of the distribution of maxima to its extreme value limit under power normalization.
\end{abstract}

MSC: Primary 62E20; 60E05; secondary 60F15; 60G15

Keywords: $P$-max stable laws; logarithmic normal distribution; maximum; uniform convergence rate

\section{Introduction}

Let $\left\{X_{n}, n \geq 1\right\}$ be a sequence of independent and identically distributed random variables with common distribution function $(d f) F(x)$. Suppose that there exist constants $a_{n}>0$, $b_{n} \in \mathbb{R}$ and a non-degenerate distribution $G(x)$ such that

$$
\lim _{n \rightarrow \infty} P\left(M_{n} \leq a_{n} x+b_{n}\right)=\lim _{n \rightarrow \infty} F^{n}\left(a_{n} x+b_{n}\right)=G(x)
$$

for all $x \in C(G)$, the set of all continuity points of $G$, where $M_{n}=\max _{1 \leq i \leq n} X_{i}$ denotes the largest of the first $n$. Then $G(x)$ must belong to one of the following three classes:

$$
\begin{aligned}
& \Phi_{\alpha}(x)= \begin{cases}0, & \text { if } x<0, \\
\exp \left\{-x^{-\alpha}\right\}, & \text { if } x \geq 0,\end{cases} \\
& \Psi_{\alpha}(x)= \begin{cases}\exp \left\{-(-x)^{\alpha}\right\}, & \text { if } x<0, \\
1, & \text { if } x \geq 0,\end{cases} \\
& \Lambda(x)=\exp \left\{-e^{-x}\right\}, \quad x \in \mathbb{R},
\end{aligned}
$$

where $\alpha$ is one positive parameter. We say that $F$ is in the max domain of attraction of $G$ if (1.1) holds, denoted by $F \in D_{l}(G)$. Criteria for $F \in D_{l}(G)$ and the choice of normalizing constants $a_{n}$ and $b_{n}$ can be found in Galambos [1], Leadbetter et al. [2], Resnick [3], and De Haan and Ferreira [4].

The limit distributions of maxima under power normalization was first derived by Pancheva [5]. A $d f F$ is said to belong to the max domain of attraction of a non-degenerate

(c) 2016 Huang et al. This article is distributed under the terms of the Creative Commons Attribution 4.0 International License (http://creativecommons.org/licenses/by/4.0/), which permits unrestricted use, distribution, and reproduction in any medium, provided you give appropriate credit to the original author(s) and the source, provide a link to the Creative Commons license, and indicate if changes were made. 
df $H$ under power normalization, written as $F \in D_{p}(H)$, if there exist constants $\alpha_{n}>0$ and $\beta_{n}>0$ such that

$$
\lim _{n \rightarrow \infty} P\left(\left|\frac{M_{n}}{\alpha_{n}}\right|^{\frac{1}{\beta_{n}}} \operatorname{sign}\left(M_{n}\right) \leq x\right)=\lim _{n \rightarrow \infty} F^{n}\left(\alpha_{n}|x|^{\beta_{n}} \operatorname{sign}(x)\right)=H(x),
$$

where $\operatorname{sign}(x)=-1,0$ or 1 according to $x<0, x=0$ or $x>0$. Pancheva [5] showed that $H$ can be only of power type of the $d f$ 's, that is,

$$
\begin{aligned}
& H_{1, \alpha}(x)= \begin{cases}0, & \text { if } x \leq 1, \\
\exp \left\{-(\log x)^{-\alpha}\right\}, & \text { if } x>1,\end{cases} \\
& H_{2, \alpha}(x)= \begin{cases}0, & \text { if } x \leq 0, \\
\exp \left\{-(-\log x)^{\alpha}\right\}, & \text { if } 0<x<1, \\
1, & \text { if } x \geq 1,\end{cases} \\
& H_{3, \alpha}(x)= \begin{cases}0, & \text { if } x \leq-1, \\
\exp \left\{-(-\log (-x))^{-\alpha}\right\}, & \text { if }-1<x<0, \\
1, & \text { if } x \geq 0,\end{cases} \\
& H_{4, \alpha}(x)= \begin{cases}\exp \left\{-(\log (-x))^{\alpha}\right\}, & \text { if } x<-1, \\
1, & \text { if } x \geq-1,\end{cases} \\
& H_{5, \alpha}(x)=\Phi_{1}(x)= \begin{cases}0, & \text { if } x \leq 0, \\
\exp \left\{-x^{-1}\right\}, & \text { if } x>0,\end{cases} \\
& H_{6, \alpha}(x)=\Psi_{1}(x)= \begin{cases}\exp \{x\}, & \text { if } x<0, \\
1, & \text { if } x \geq 0,\end{cases}
\end{aligned}
$$

where $\alpha$ is a positive parameter. Necessary and sufficient conditions for $F$ to satisfy (1.2) have been given by Christoph and Falk [6], Mohan and Ravi [7], Mohan and Subramanya [8] and Subramanya [9].

The logarithmic normal distribution (lognormal distribution for short) is one of the most widely applied distributions in statistics, biology, and some other disciplines. In this paper, we are interested in considering the uniform rate of convergence of (1.2) with $X_{n}$ following the lognormal distribution. The probability density function of the lognormal distribution is given by

$$
F^{\prime}(x)=\frac{x^{-1}}{\sqrt{2 \pi}} \exp \left\{-\frac{(\log x)^{2}}{2}\right\}, \quad x>0
$$

One interesting problem in extreme value analysis is to estimate the rate of uniform convergence of $F^{n}(\cdot)$ to its extreme value distribution. For a power normalization, Chen et $a l$. [10] derived the convergence rates of the distribution of maxima for random variables obeying the general error distribution. For convergence rates of distributions of extremes under linear normalization, see De Haan and Resnick [11] under second-order regular 
variation and for special cases see Hall [12] and Nair [13] for the normal distribution, which also is extended to those such as general error distribution, logarithmic general error distribution, see recent work of Peng et al. [14] and Liao and Peng [15]. For other related work on the convergence rates of some given distributions, see Castro [16] for the gamma distribution, Lin et al. [17] for the short-tailed symmetric distribution due to Tiku and Vaughan [18], and Liao et al. [19] for the skew normal distribution which extended the results of Nair [13]. The aim of this paper is to study the uniform and point-wise convergence rates of the distribution of power normalized maxima to its limits, respectively.

The contents of this article is organized as follows: some auxiliary results are given in Section 2. In Section 3, we provide our main results with related proofs deferred to Section 4 .

\section{Preliminaries}

To prove our results, we first cite some results from Liao and Peng [15] and Mohan and Ravi [7].

In the sequel, let $\left\{X_{n}, n \geq 1\right\}$ be a sequence of independent identically distributed random variables with common $d f F$ which follows the lognormal distribution. As before, let $M_{n}=$ $\max _{1 \leq i \leq n} X_{i}$ represent the partial maximum of $\left\{X_{n}, n \geq 1\right\}$. Liao and Peng [15] defined

$$
a_{n}=\frac{\exp \left((2 \log n)^{1 / 2}\right)}{(2 \log n)^{1 / 2}}, \quad b_{n}=\left(\exp \left((2 \log n)^{1 / 2}\right)\right)\left(1-\frac{\log 4 \pi+\log \log n}{2(2 \log n)^{1 / 2}}\right),
$$

and they obtained

$$
\lim _{n \rightarrow \infty} P\left(\left(M_{n}-b_{n}\right) / a_{n} \leq x\right)=\exp \left(-e^{-x}\right)=: \Lambda(x) .
$$

From (2.2) we immediately derive $F \in D_{l}(\Lambda)$. The following Mills ratio of the lognormal distribution is due to Liao and Peng [15]:

$$
\frac{1-F(x)}{F^{\prime}(x)} \sim \frac{x}{\log x},
$$

as $x \rightarrow \infty$, where $F^{\prime}(x)$ is the density function of the lognormal distribution $F(x)$. According to Liao and Peng [15], we have

$$
1-F(x)=c(x) \exp \left(-\int_{e}^{x} \frac{g(t)}{f(t)} \mathrm{d} t\right)
$$

for sufficiently large $x$, where $c(x) \rightarrow(2 \pi e)^{-1 / 2}$ as $x \rightarrow \infty, g(x)=1+(\log x)^{-2}$ and

$$
f(x)=\frac{x}{\log x} .
$$

Note that $f^{\prime}(x) \rightarrow 0$ and $g(x) \rightarrow 1$ as $x \rightarrow \infty$.

Lemma 2.1 [15] Let $F$ denote the lognormal distribution function. Then

$$
\begin{aligned}
1-F(x) & =\frac{1}{\sqrt{2 \pi}}(\log x)^{-1} \exp \left(-\frac{(\log x)^{2}}{2}\right)-\gamma(x) \\
& =\frac{1}{\sqrt{2 \pi}}(\log x)^{-1} \exp \left(-\frac{(\log x)^{2}}{2}\right)\left(1-(\log x)^{-2}\right)+\mathcal{S}(x)
\end{aligned}
$$


for $x>1$, where

$$
0<\gamma(x)<\frac{1}{\sqrt{2 \pi}}(\log x)^{-3} \exp \left(-\frac{(\log x)^{2}}{2}\right)
$$

and

$$
0<\mathcal{S}(x)<\frac{3}{\sqrt{2 \pi}}(\log x)^{-5} \exp \left(-\frac{(\log x)^{2}}{2}\right) .
$$

In order to obtain the main results, we need the following two lemmas.

Lemma 2.2 [7] Let $F$ denote a $d f$ and $r(F)=\sup \{x: F(x)<1\}$. Suppose that $F \in D_{l}(\Lambda)$ and $r(F)=\infty$, then $F \in D_{p}\left(\Phi_{1}\right)$, where normalizing constants $\alpha_{n}=b_{n}, \beta_{n}=a_{n} / b_{n}$.

Lemma 2.3 [7] Let $F$ denote a $d f$, if $F \in D_{p}\left(\Phi_{1}\right)$ if and only if

(i) $r(F)>0$, and

(ii) $\lim _{t \uparrow r(F)} \frac{1-F(\operatorname{texp}(\bar{y}(t)))}{1-F(t)}=e^{-y}$, for some positive valued function $\bar{f}$.

If (ii) holds for some $\bar{f}$, then $\int_{a}^{r(F)}((1-F(x)) / x) \mathrm{d} x<\infty$ for $0<a<r(F)$ and (ii) holds with the choice $\bar{f}(t)=\int_{t}^{r(F)}((1-F(x)) / x) \mathrm{d} x /(1-F(t))$. The normalizing constants may be chosen as $\alpha_{n}=F^{\leftarrow}(1-1 / n)$ and $\beta_{n}=\bar{f}\left(\alpha_{n}\right)$, where $F^{\leftarrow}(x)=\inf \{y: F(y) \geq x\}$.

Theorem 2.1 Let $\left\{X_{n}, n \geq 1\right\}$ be a sequence of independent identically distributed lognormal random variables. Then $F \in D_{p}\left(\Phi_{1}\right)$ and the normalizing constants can be chosen as $\alpha_{n}^{*}=b_{n}, \beta_{n}^{*}=a_{n} / b_{n}$, where $a_{n}$ and $b_{n}$ are given by (2.1).

Proof Note that $F$ follows the lognormal distribution, which implies $F \in D_{p}\left(\Phi_{1}\right)$ and $\alpha_{n}^{*}=$ $b_{n}, \beta_{n}^{*}=a_{n} / b_{n}$ by Lemma 2.2 , where $a_{n}$ and $b_{n}$ are defined by (2.1).

By Lemma 2.3 and (2.3) and combining with Proposition 1.1(a) in [3], a natural way to choose constants $\alpha_{n}$ and $\beta_{n}$ is to solve the following equations:

$$
2 \pi\left(\log \alpha_{n}\right)^{2} \exp \left(\left(\log \alpha_{n}\right)^{2}\right)=n^{2}
$$

and

$$
\beta_{n}=\frac{f\left(\alpha_{n}\right)}{\alpha_{n}}=\frac{1}{\log \alpha_{n}},
$$

where $f$ is given by (2.4). The solution of (2.9) may be expressed as

$$
\alpha_{n}=\left(\exp \left((2 \log n)^{1 / 2}\right)\right)\left(1-\frac{\log 4 \pi+\log \log n}{2(2 \log n)^{1 / 2}}+o\left(\frac{1}{(\log n)^{1 / 2}}\right)\right)
$$

and we easily check that $\beta_{n} \sim(2 \log n)^{-1 / 2}$.

\section{Main results}

In this section, we give two main results. Theorem 3.1 proves the result that the rate of uniform convergence of $F^{n}\left(\alpha_{n} x^{\beta_{n}}\right)$ to its extreme value limit is proportional to $1 / \log n$. Theorem 3.2 establishes the result that the point-wise rate of convergence of $\left|M_{n} / \alpha_{n}\right|^{1 / \beta_{n}} \operatorname{sign}\left(M_{n}\right)$ to the extreme value $d f \exp \left(-x^{-1}\right)$ is of the order of $O\left(x^{-1}(\log x)^{2} \times\right.$ $\left.e^{-1 / x}(\log n)^{-1}\right)$. 
Theorem 3.1 Let $\left\{X_{n}, n \geq 1\right\}$ denote an independent identically distributed random variables sequence with common $d f F$ following the lognormal distribution. Then there exist absolute constants $0<\mathcal{C}_{1}<\mathcal{C}_{2}$ such that

$$
\frac{\mathcal{C}_{1}}{\log n}<\sup _{x>0}\left|F^{n}\left(\alpha_{n} x^{\beta_{n}}\right)-\Phi_{1}(x)\right|<\frac{\mathcal{C}_{2}}{\log n}
$$

for large $n>n_{0}$, where $\alpha_{n}$ and $\beta_{n}$ are determined by (2.9) and (2.10), respectively.

Theorem 3.2 Let $\alpha_{n}$ and $\beta_{n}$ be given by (2.9) and (2.10). Then, for fixed $x>0$,

$$
\left|F^{n}\left(\alpha_{n} x^{\beta_{n}}\right)-\Phi_{1}(x)\right| \sim x^{-1} e^{-1 / x}\left(1+\left(1+\frac{1}{2} \log x\right) \log x\right) \frac{1}{2 \log n},
$$

as $n \rightarrow \infty$.

\section{Proofs}

First of all, we provide the proof of Theorem 3.2, for it is relatively easy.

Proof of Theorem 3.2 By Lemma 2.1, we have

$$
\begin{aligned}
1-F\left(\alpha_{n} x^{\beta_{n}}\right)= & \frac{1}{\sqrt{2 \pi}}\left(\log \left(\alpha_{n} x^{\beta_{n}}\right)\right)^{-1} \exp \left(-\frac{\left(\log \left(\alpha_{n} x^{\beta_{n}}\right)\right)^{2}}{2}\right) \\
& \times\left(1-\left(\log \left(\alpha_{n} x^{\beta_{n}}\right)\right)^{-2}\right)+\mathcal{S}\left(\alpha_{n} x^{\beta_{n}}\right) \\
=: & T_{1}(x) T_{2}(x)+T_{3}(x)
\end{aligned}
$$

for $x>0$, where $T_{1}(x)=\frac{1}{\sqrt{2 \pi}}\left(\log \left(\alpha_{n} x^{\beta_{n}}\right)\right)^{-1} \exp \left(-\frac{\left(\log \left(\alpha_{n} x^{\beta_{n}}\right)\right)^{2}}{2}\right), T_{2}(x)=1-\left(\log \left(\alpha_{n} x^{\beta_{n}}\right)\right)^{-2}$ and $T_{3}(x)=\mathcal{S}\left(\alpha_{n} x^{\beta_{n}}\right)$.

First, we calculate $T_{1}(x)$. By (2.9) and (2.10), we have

$$
\begin{aligned}
T_{1}(x)= & \frac{1}{\sqrt{2 \pi}}\left(\log \alpha_{n}\right)^{-1} \exp \left(-\frac{\left(\log \alpha_{n}\right)^{2}}{2}\right)\left(1+\left(\log \alpha_{n}\right)^{-1} \beta_{n} \log x\right)^{-1} \\
& \times \exp \left(-\left(\log \alpha_{n}\right) \beta_{n} \log x-\frac{\beta_{n}^{2} \log ^{2} x}{2}\right) \\
= & \frac{1}{n x}\left(1+\beta_{n}^{2} \log x\right)^{-1} \exp \left(-\frac{\beta_{n}^{2} \log ^{2} x}{2}\right) \\
= & \frac{1}{n x}\left(1-\beta_{n}^{2} \log x+O\left(\beta_{n}^{4}\right)\right)\left(1-\frac{\beta_{n}^{2} \log ^{2} x}{2}+O\left(\beta_{n}^{4}\right)\right) \\
= & \frac{1}{n x}\left(1-\beta_{n}^{2}\left(1+\frac{1}{2} \log x\right) \log x+O\left(\beta_{n}^{4}\right)\right) .
\end{aligned}
$$

Second, we estimate $T_{2}(x)$ and $T_{3}(x)$ for $x>0$. By (2.10), we derive

$$
\begin{aligned}
T_{2}(x) & =1-\beta_{n}^{2}\left(1+\beta_{n}^{2} \log x\right)^{-2} \\
& =1-\beta_{n}^{2}\left(1-2 \beta_{n}^{2} \log x+O\left(\beta_{n}^{4}\right)\right) \\
& =1-\beta_{n}^{2}+O\left(\beta_{n}^{4}\right),
\end{aligned}
$$


and by Lemma 2.1 we have

$$
\begin{aligned}
T_{3}(x) & \leq \frac{3}{\sqrt{2 \pi}}\left(\log \left(\alpha_{n} x^{\beta_{n}}\right)\right)^{-5} \exp \left(-\frac{\left(\log \left(\alpha_{n} x^{\beta_{n}}\right)\right)^{2}}{2}\right) \\
& =3 \beta_{n}^{4}\left(1+\beta_{n}^{2} \log x\right)^{-4} T_{1}(x) \\
& =O\left(n^{-1} \beta_{n}^{4}\right) .
\end{aligned}
$$

By (4.1)-(4.3), we have

$$
1-F^{n}\left(\alpha_{n} x^{\beta_{n}}\right)=\frac{1}{n x}\left(1-\beta_{n}^{2}\left(1+\left(1+\frac{1}{2} \log x\right) \log x\right)+O\left(\beta_{n}^{4}\right)\right) .
$$

Thus, we obtain

$$
\begin{aligned}
& F^{n}\left(\alpha_{n} x^{\beta_{n}}\right)-\Phi_{1}(x) \\
& \quad=\left(1-\frac{1}{n x}\left(1-\beta_{n}^{2}\left(1+\left(1+\frac{1}{2} \log x\right) \log x\right)+O\left(\beta_{n}^{4}\right)\right)\right)^{n}-\exp \left(-\frac{1}{x}\right) \\
& \quad=\exp \left(-\frac{1}{x}\right)\left(\exp \left(\frac{1}{x}\left(\beta_{n}^{2}\left(1+\left(1+\frac{1}{2} \log x\right) \log x\right)+O\left(\beta_{n}^{4}\right)\right)\right)-1\right) \\
& \quad=\exp \left(-\frac{1}{x}\right)\left(\beta_{n}^{2} \frac{1}{x}\left(1+\left(1+\frac{1}{2} \log x\right) \log x\right)+O\left(\beta_{n}^{4}\right)\right)
\end{aligned}
$$

for large $n$ and $x>0$. We immediately get the result of Theorem 3.2 by (4.4).

Proof of Theorem 3.1 By Theorem 3.2 we can prove that there exists an absolute constant $\mathcal{C}_{1}$ such that

$$
\sup _{x>0}\left|F^{n}\left(\alpha_{n} x^{\beta_{n}}\right)-\Phi_{1}(x)\right|>\frac{\mathcal{C}_{1}}{\log n} .
$$

In order to obtain the upper bound for $x>0$, we need to prove
(a) $\sup _{1 \leq x<\infty}\left|F^{n}\left(\alpha_{n} x^{\beta_{n}}\right)-\Phi_{1}(x)\right|<d_{1} \beta_{n}^{2}$,
(b) $\sup _{c_{n} \leq x<1}\left|F^{n}\left(\alpha_{n} x^{\beta_{n}}\right)-\Phi_{1}(x)\right|<d_{2} \beta_{n}^{2}$,
(c) $\sup _{0<x<c_{n}}\left|F^{n}\left(\alpha_{n} x^{\beta_{n}}\right)-\Phi_{1}(x)\right|<d_{3} \beta_{n}^{2}$

for $n>n_{0}$, where $d_{i}>0, i=1,2,3$ are absolute constants and

$$
c_{n}=\frac{1}{2 \log \log \alpha_{n}}
$$

is positive for $n>n_{0}$. By (2.9), we have

$$
0.4(2 \log n)^{1 / 2}<\log \alpha_{n}<(2 \log n)^{1 / 2}
$$

for $n>n_{0}$. 
First, consider the case of $x \geq c_{n}$. Set

$$
\begin{aligned}
& R_{n}(x)=-\left[n \log F\left(\alpha_{n} x^{\beta_{n}}\right)+n \Psi_{n}(x)\right], \\
& B_{n}(x)=\exp \left(-R_{n}\right), \quad A_{n}(x)=\exp \left(-n \Psi_{n}(x)+\frac{1}{x}\right),
\end{aligned}
$$

where $\Psi_{n}(x)=1-F\left(\alpha_{n} x^{\beta_{n}}\right)$ and $A_{n}(x) \rightarrow 1$, as $x \rightarrow \infty$. We have

$$
\begin{aligned}
\Psi_{n}(x) & \leq \Psi_{n}\left(c_{n}\right)<\frac{1}{\sqrt{2 \pi}}\left(\log \left(\alpha_{n} c_{n}^{\beta_{n}}\right)\right)^{-1} \exp \left(-\frac{\left(\log \left(\alpha_{n} c_{n}^{\beta_{n}}\right)\right)^{2}}{2}\right) \\
& =\frac{1}{n}\left(1+\beta_{n}^{2} \log c_{n}\right)^{-1} \exp \left(-\log c_{n}-\frac{\beta_{n}^{2} \log ^{2} c_{n}}{2}\right) \\
& <\frac{1}{n}\left(1+\beta_{n}^{2} \log c_{n}\right)^{-1} c_{n}^{-1} \\
& =\left(1-\frac{\log \left(2 \log \log \alpha_{n}\right)}{\left(\log \alpha_{n}\right)^{2}}\right)^{-1} \frac{2 \log \log \alpha_{n}}{n} \\
& <\tilde{c}_{4}<1
\end{aligned}
$$

for $n>n_{0}$. So,

$$
\inf _{x>c_{n}}\left(1-\Psi_{n}(x)\right)>1-\tilde{c}_{4}>0 .
$$

Since

$$
-x-\frac{x^{2}}{2(1-x)}<\log (1-x)<-x
$$

for $0<x<1$, we obtain

$$
\begin{aligned}
0 & <R_{n}(x) \leq \frac{n \Psi_{n}^{2}(x)}{2\left(1-\Psi_{n}(x)\right)}<\frac{n \Psi_{n}^{2}\left(c_{n}\right)}{2\left(1-\Psi_{n}(x)\right)} \\
& <\frac{n^{-1}\left(1+\beta_{n}^{2} \log c_{n}\right)^{-2} c_{n}^{-2}}{2\left(1-\Psi_{n}(x)\right)} \\
& <\frac{n^{-1}\left(1+\beta_{n}^{2} \log c_{n}\right)^{-2} c_{n}^{-2}\left(\log \alpha_{n}\right)^{2}}{2\left(1-\tilde{c}_{4}\right) \beta_{n}^{-2}} \\
& =\frac{2}{\sqrt{2 \pi}\left(1-\tilde{c}_{4}\right)}\left(1-\frac{\log \left(2 \log \log \alpha_{n}\right)}{\left(\log \alpha_{n}\right)^{2}}\right)^{-2} \frac{\left(\log \log \alpha_{n}\right)^{2} \log \alpha_{n}}{\exp \left(\frac{\left(\log \alpha_{n}\right)^{2}}{2}\right)} \beta_{n}^{2} \\
& <\tilde{c}_{5} \beta_{n}^{2}
\end{aligned}
$$

for $n>n_{0}$.

Hence, we have

$$
n^{-1} \beta_{n}^{-2}\left(1+\beta_{n}^{2} \log c_{n}\right)^{-2} c_{n}^{-2}<\tilde{c}_{6}
$$

for $n>n_{0}$. Thus,

$$
\left|B_{n}(x)-1\right|<R_{n}<\tilde{c}_{5} \beta_{n}^{2}
$$


for $n>n_{0}$. By (4.8), we have

$$
\begin{aligned}
& \left|F^{n}\left(\alpha_{n} x^{\beta_{n}}\right)-\Phi_{1}(x)\right| \\
& \quad \leq \Phi_{1}(x) B_{n}(x)\left|A_{n}(x)-1\right|+\left|B_{n}(x)-1\right| \\
& \quad<\Phi_{1}(x)\left|A_{n}(x)-1\right|+\tilde{c}_{5} \beta_{n}^{2}
\end{aligned}
$$

for $x \geq c_{n}$.

We now prove (4.5). By (2.9), (2.10), and the definition of $A_{n}(x)$, we have

$$
A_{n}^{\prime}(x)=A_{n}(x) \frac{1}{x^{2}}\left(\exp \left(-\frac{1}{2} \beta_{n}^{2} \log ^{2} x\right)-1\right)<0
$$

for $x>1$. Since

$$
\begin{aligned}
& 0<n \gamma\left(\alpha_{n}\right)<\beta_{n}^{2} \text { and } e^{x}-1 \leq x e^{x} \quad \text { for } 0 \leq x \leq 1 \text { and } \\
& \exp \left(n \gamma\left(\alpha_{n}\right)\right)<\exp \left(\beta_{n}^{2}\right)<\exp \left(\frac{25}{8 \log n}\right)<\exp \left(\frac{25}{8 \log n_{0}}\right) \text { for } n>n_{0}
\end{aligned}
$$

and by (2.5), (2.9), we have

$$
\begin{aligned}
\sup _{x \geq 1}\left|A_{n}(x)-1\right| & =\left|A_{n}(1)-1\right| \\
& =\left|\exp \left(n \gamma\left(\alpha_{n}\right)\right)-1\right| \\
& \leq n \gamma\left(\alpha_{n}\right) \exp \left(n \gamma\left(\alpha_{n}\right)\right) \\
& \leq \tilde{c}_{7} \beta_{n}^{2}
\end{aligned}
$$

for $n>n_{0}$.

Combining (4.9) with (4.10), we have

$$
\sup _{x \geq 1}\left|F^{n}\left(\alpha_{n} x^{\beta_{n}}\right)-\Phi_{1}(x)\right|<\left(\tilde{c}_{5}+\tilde{c}_{7}\right) \beta_{n}^{2}
$$

Second, consider the situation of $c_{n} \leq x<1$. By Lemma 2.1, we obtain

$$
\begin{aligned}
-n \Psi_{n}(x)+\frac{1}{x}= & -n\left(\frac{1}{\sqrt{2 \pi}}\left(\log \left(\alpha_{n} x^{\beta_{n}}\right)\right)^{-1} \exp \left(-\frac{\left(\log \left(\alpha_{n} x^{\beta_{n}}\right)\right)^{2}}{2}\right)-\gamma\left(\alpha_{n} x^{\beta_{n}}\right)\right)+\frac{1}{x} \\
= & -n\left(\frac{1}{\sqrt{2 \pi}}\left(\log \left(\alpha_{n} x^{\beta_{n}}\right)\right)^{-1} \exp \left(-\frac{\left(\log \left(\alpha_{n} x^{\beta_{n}}\right)\right)^{2}}{2}\right)\right. \\
& \left.-\frac{1}{\sqrt{2 \pi}}\left(\log \left(\alpha_{n} x^{\beta_{n}}\right)\right)^{-3} q_{n}\left(\alpha_{n} x^{\beta_{n}}\right) \exp \left(-\frac{\left(\log \left(\alpha_{n} x^{\beta_{n}}\right)\right)^{2}}{2}\right)\right)+\frac{1}{x} \\
= & \frac{1}{x}\left(1+\beta_{n}^{2} \log x\right)^{-1}\left(-\left(1-\left(\log \alpha_{n}\right)^{-2} q_{n}\left(\alpha_{n} x^{\beta_{n}}\right)\left(1+\beta_{n}^{2} \log x\right)^{-2}\right)\right. \\
& \left.\times \exp \left(-\frac{1}{2} \beta_{n}^{2} \log ^{2} x\right)+1+\beta_{n}^{2} \log x\right) \\
= & \frac{1}{x}\left(1+\beta_{n}^{2} \log x\right)^{-1} Q_{n}(x),
\end{aligned}
$$


where $0<q_{n}(x)<1$ and

$$
Q_{n}(x)=-\left(1-\beta_{n}^{2} q_{n}\left(\alpha_{n} x^{\beta_{n}}\right)\left(1+\beta_{n}^{2} \log x\right)^{-2}\right) \exp \left(-\frac{1}{2} \beta_{n}^{2} \log ^{2} x\right)+1+\beta_{n}^{2} \log x .
$$

Since $e^{-x}>1-x$, as $x>0$, we have

$$
\begin{aligned}
Q_{n}(x) & <-\left(1-\beta_{n}^{2} q_{n}\left(\alpha_{n} x^{\beta_{n}}\right)\left(1+\beta_{n}^{2} \log x\right)^{-2}\right)\left(1-\frac{1}{2} \beta_{n}^{2} \log ^{2} x\right)+1+\beta_{n}^{2} \log x \\
& <\beta_{n}^{2}\left(\left(1+\beta_{n}^{2} \log x\right)^{-2}+\frac{1}{2} \log ^{2} x\right) .
\end{aligned}
$$

But

$$
\begin{aligned}
Q_{n}(x) & >\beta_{n}^{2} q_{n}\left(\alpha_{n} x^{\beta_{n}}\right)\left(1+\beta_{n}^{2} \log x\right)^{-2}+\beta_{n}^{2} \log x \\
& >\beta_{n}^{2} \log x .
\end{aligned}
$$

Hence, we obtain

$$
\begin{aligned}
\left|Q_{n}(x)\right| & <\beta_{n}^{2}\left(\left(1+\beta_{n}^{2} \log x\right)^{-2}+\frac{1}{2} \log ^{2} x+|\log x|\right) \\
& <\beta_{n}^{2}\left(\left(1-\frac{\log \left(2 \log \log \alpha_{n}\right)}{\log ^{2} \alpha_{n}}\right)^{-2}+\frac{1}{2} \log ^{2} x+|\log x|\right) \\
& <\beta_{n}^{2}\left(\tilde{c}_{8}+\frac{1}{2} \log ^{2} x+|\log x|\right)
\end{aligned}
$$

for $n>n_{0}$, where $c_{n} \leq x<1$. Therefore,

$$
\begin{aligned}
\left|-n \Psi_{n}(x)+\frac{1}{x}\right| & <\beta_{n}^{2}\left(\tilde{c}_{8}+\frac{1}{2} \log ^{2} x+|\log x|\right) x^{-1}\left(1+\beta_{n}^{2} \log x\right)^{-1} \\
& <\beta_{n}^{2}\left(\tilde{c}_{8}+\frac{1}{2} \log ^{2} c_{n}+\left|\log c_{n}\right|\right) c_{n}^{-1}\left(1+\beta_{n}^{2} \log c_{n}\right)^{-1} \\
& <\tilde{c}_{9}
\end{aligned}
$$

for $n \geq n_{0}$. Thus, there exists a positive number $\theta$ satisfying $0<\theta<1$ such that

$$
\begin{aligned}
\Phi_{1}(x)\left|A_{n}(x)-1\right| & <\Phi_{1}(x) \exp \left(\theta\left(-n \Psi_{n}(x)+\frac{1}{x}\right)\right)\left|-n \Psi_{n}(x)+\frac{1}{x}\right| \\
& <\exp \left(\tilde{c}_{9}\right) \beta_{n}^{2} \sup _{c_{n} \leq x<1}\left|\left(\tilde{c}_{8}+\frac{1}{2} \log ^{2} x+|\log x|\right) x^{-1}\right|\left(1+\beta_{n}^{2} \log c_{n}\right)^{-1} \\
& <\tilde{c}_{10} \beta_{n}^{2} .
\end{aligned}
$$

By (4.9) and (4.11), the proof of (4.6) is complete.

Third, consider the circumstance of $0<x<c_{n}$. In this case

$$
\Phi_{1}(x)<\Phi_{1}\left(c_{n}\right)=\beta_{n}^{2},
$$


we have

$$
\begin{aligned}
\sup _{0<x<c_{n}}\left|F^{n}\left(\alpha_{n} x^{\beta_{n}}\right)-\Phi_{1}(x)\right| & <F^{n}\left(\alpha_{n} c_{n}^{\beta_{n}}\right)+\Phi_{1}\left(c_{n}\right) \\
& <\sup _{c_{n}<x<1}\left|F^{n}\left(\alpha_{n} x^{\beta_{n}}\right)-\Phi_{1}(x)\right|+2 \Phi_{1}\left(c_{n}\right) \\
& <\left(\tilde{c}_{5}+\tilde{c}_{10}\right) \beta_{n}^{2}+\beta_{n}^{2} \\
& <\tilde{c}_{11} \beta_{n}^{2} .
\end{aligned}
$$

The proof of Theorem 3.1 is finished.

\section{Competing interests}

The authors declare that they have no competing interests.

\section{Authors' contributions}

$\mathrm{JH}$ obtained the theorem and completed the proof. SC and YL corrected and improved the final version. All authors read and approved the final manuscript.

\section{Author details}

${ }^{1}$ School of Mathematics and Computational Science, Zunyi Normal College, Zunyi, 563002, China. ${ }^{2}$ School of Mathematics and Statistics, Southwest University, Chongqing, 400715, China.

\section{Acknowledgements}

The authors would like to thank the Editor-in-Chief, the Associate Editor, and the referees for carefully reading the paper and for their comments, which greatly improved the paper. This work was supported by the National Natural Science Foundation of China (Grant No. 11171275, No. 71461027), the SWU Grant for Statistics PhD, the Science and Technology fund Project of GZ (Grant No. LKZS[2014]29), the Science and Technology Plan Project of Guizhou Province (Grant No. LH[2015]7001, No. LH[2015]7055), 2013, 2014, and 2015, and the Zunyi 15851 talents elite project funding and Zhunyi innovative talent team (Zunyi KH(2015)38)

Received: 18 March 2015 Accepted: 1 February 2016 Published online: 13 February 2016

\section{References}

1. Galambos, J: The Asymptotic Theory of Extreme Order Statistics. Wiley, New York (1987)

2. Leadbetter, MR, Lindgren, G, Rootzén, H: Extremes and Related Properties of Random Sequences and Processes. Springer, New York (1983)

3. Resnick, SI: Extreme Value, Regular Variation and Point Processes. Springer, New York (1987)

4. De Haan, L, Ferreira, A: Extreme Value Theory: An Introduction. Springer, New York (2006)

5. Pancheva, E: Limit theorems for extreme order statistics under nonlinear normalization. In: Stability Problems for Stochastic Models. Lect. Notes Math., vol. 1155, pp. 284-309. Springer, Berlin (1985)

6. Christoph, G, Falk, M: A note on domains of attraction of p-max stable laws. Stat. Probab. Lett. 28, 279-284 (1996)

7. Mohan, NR, Ravi, S: Max domains of attraction of univariate and multivariate $p$-max stable laws. Theory Probab. Appl. 37, 632-643 (1993)

8. Mohan, NR, Subramanya, UR: Characterization of max domains of attraction of univariate $p$-max stable laws. In: Proceedings of the Symposium on Distribution Theory, Kochi, Kerala, India, pp. 11-24 (1991)

9. Subramanya, UR: On max domains of attraction of univariate $p$-max stable laws. Stat. Probab. Lett. 19, 271-279 (1994)

10. Chen, S, Wang, C, Zhang, G: Rates of convergence of extremes for general error distribution under power normalization. Stat. Probab. Lett. 82, 385-395 (2012)

11. De Haan, L, Resnick, SI: Second-order regular variation and rates of convergence in extreme value theory. Ann. Probab. 24, 97-124 (1996)

12. Hall, P: On the rate of convergence of normal extremes. J. Appl. Probab. 16, 433-439 (1979)

13. Nair, KA: Asymptotic distribution and moments of normal extremes. Ann. Probab. 9, 150-153 (1981)

14. Peng, Z, Nadarajah, S, Lin, F: Convergence rate of extremes for the general error distribution. J. Appl. Probab. 47, 668-679 (2010)

15. Liao, X, Peng, Z: Convergence rates of limit distribution of maxima of lognormal samples. J. Math. Anal. Appl. 395 643-653 (2012)

16. Castro, LCE: Uniform rate of convergence in extreme-value theory: normal and gamma models. Ann. Sci. Univ. Clermont-Ferrand II, Probab. Appl. 6, 25-41 (1987)

17. Lin, F, Zhang, X, Peng, Z, Jiang, Y: On the rate of convergence of STSD extremes. Commun. Stat., Theory Methods 40 1795-1806 (2011)

18. Tiku, ML, Vaughan, DC: A family of short-tailed symmetric distributions. Technical report, McMaster University, Canada (1999)

19. Liao, X, Peng, Z, Nadarajah, S, Wang, X: Rates of convergence of extremes from skew normal sample. Stat. Probab. Lett. 84, 40-47 (2014) 